\title{
Efecto del hierro en el crecimiento y acumulación de lípidos en la microalga colombiana Chlorella Vulgaris LAUN 0019
}

\author{
Luis Miguel Serrano Bermúdez \\ Estudiante MSc. Ingeniería Química, \\ Investigador Grupo Procesos Químicos y Bioquímicos, \\ Universidad Nacional, Bogotá, Colombia \\ Imserranob@unal.edu.co \\ Édwar Rodrigo Sierra Sáenz \\ Estudiante MSc. Ingeniería Química, \\ Universidad Nacional, Bogotá, Colombia \\ ersierras@unal.edu.co \\ Carlos Andrés Álvarez Sierra \\ Ingeniero Químico \\ Universidad Nacional, Bogotá, Colombia \\ caalvarezs@unal.edu.co \\ Paulo César Narváez Rincón \\ Ph. D. en Ingeniería Química. \\ Universidad Nacional, Bogotá, Colombia \\ Docente Tiempo Completo, Investigador Grupo Procesos Quími- \\ cos y Bioquímicos, Universidad Nacional, Bogotá, Colombia \\ pcnarvaez@unal.edu.co
}

Resumen-En este trabajo se evalúa el efecto del ión ferroso (Fe2+) sobre el crecimiento y acumulación de lípidos totales de la microalga Chlorella vulgaris. Se empleó medio Bristol estándar para su cultivo; la cinética de crecimiento se midió por conteo directo y la determinación de lípidos totales se realizó mediante extracción con Soxhlet. Se estudiaron cinco diferentes concentraciones de este ión, entre 2,16 $\mu \mathrm{M}$ y 50,0 $\mu \mathrm{M}$. El medio enriquecido con una concentración de $\mathbf{1 0 , 0} \mu \mathrm{M}$ produjo la máxima velocidad específica de crecimiento celular (0,76 día-1), mientras que las máximas productividades de biomasa y de lípidos se presentaron a la concentración 5,00 $\mu \mathrm{M}$ con valores de 112,4 mg-L-1-día-1 y 6,52 mg-L-1-día-1 respectivamente. Para las concentraciones más altas de hierro (21,5 y 50,0 $\mu \mathrm{M})$, la microalga presentó inhibición por sustrato. Finalmente, para concentraciones menores que 10,0 $\mu \mathrm{M}$ se encontró que para una significancia del $\mathbf{5} \%$ la concentración del hierro no afecta significativamente la productividad de biomasa y lípidos.

Palabras clave- Chlorella vulgaris, hierro, lípidos, medio Bristol, microalga.

Abstract- This paper describes the evaluation of the effect of ferrous iron (Fe2+) on the growth and accumulation of total lipids on the microalgae Chlorella vulgaris. Standard Bristol Medium was used for cultivation. Growth kinetics was measured by direct counting while total lipids determination was performed by Soxhlet extraction. Five different concentrations of Fe2+, between $2.16 \mu \mathrm{M}$ and $50.0 \mu \mathrm{M}$ were studied. The medium enriched at a concentration of $\mathbf{1 0 . 0} \mu \mathrm{M}$ produced the maximum specific cell growth rate (0.76 day-1), while the maximum productivities of biomass and lipid concentration were obtained at $5.00 \mu \mathrm{M}$, with values of
Daniel Mauricio Ramírez Landínez

\author{
Estudiante MSc. Ingeniería Química, Investigador Grupo \\ Procesos Químicos y Bioquímicos, Universidad Nacional, \\ Bogotá, Colombia \\ dramirezl@unal.edu.co \\ Ómar Mauricio Scott Carvajal \\ Estudiante MSc. Ingeniería Química, \\ Universidad Nacional, Bogotá, Colombia \\ omscottc@unal.edu.co \\ Juan Manuel Torres Parra \\ Ingeniero Químico \\ Universidad Nacional, Bogotá, Colombia \\ jmtorresp@unal.edu.co \\ Rubén Darío Godoy Silva \\ Ph. D. en Ingeniería Química. \\ The Ohio State University. Columbus, Ohio. USA \\ Docente Tiempo Completo, Investigador Grupo Procesos \\ Químicos y Bioquímicos, Universidad Nacional, \\ Bogotá, Colombia \\ rdgodoys@bt.unal.edu.co
}

112.4 mg-L-1-day-1 and 6.52 mg-L-1-day-1, respectively. For higher concentrations of iron (21.5 and $50.0 \mu \mathrm{M})$, the microalgae showed substrate inhibition. Finally, for concentrations lower than $\mathbf{1 0 . 0} \mu \mathrm{M}$, it was found that for a statistical significance of $5 \%$ iron concentration does not significantly affect the productivity of biomass and lipids.

Keywords- Chlorella vulgaris, Iron, Lipids, Bristol Medium, Microalgae.

\section{INTRODUCCIÓN}

Existe una gran controversia en torno a los beneficios reales de los ésteres metílicos obtenidos a partir de los triglicéridos presentes en los aceites vegetales y usados actualmente como combustible (biodiesel); la controversia abarca tanto los aspectos ambientales como los sociales, especialmente por el desplazamiento de terrenos y cultivos dedicados tradicionalmente a surtir alimentos [1], [2]. Tales cultivos incluyen soya, colza, palma de aceite y maíz [3]-[6]. Adicionalmente, debido a las limitaciones en cuanto al área cultivada de estas plantas, el desarrollo a escala industrial de la producción de biodiesel no puede, en la realidad, satisfacer más que una pequeña parte de la actual demanda de combustibles [7].

Muchas especies de microalgas acumulan durante su crecimiento significativas cantidades de lípidos que pueden extraerse y destinarse a usos 
diversos de acuerdo con su composición [7], [8]. Dentro de la enorme variedad de lípidos producidos por las microalgas, se destacan los triglicéridos de cadenas saturadas y poliinsaturadas, lípidos variados de alto peso molecular (hasta $\mathrm{C}_{40}$ ) y ácidos grasos libres (como los ácidos eicosapentanoico, linolénico y docosahexanoico) de alto vaIor nutricional y farmacéutico [9]. Los triglicéridos, por ejemplo, pueden transformarse en biodiesel, producto que ha cobrado importancia en los últimos años como consecuencia de la incertidumbre en el precio y las reservas del petróleo y la búsqueda de fuentes alternativas de energía [10].

Entre las microalgas que son capaces de acumular grandes cantidades de aceite destacan Botryococcus braunii, Nannochloropsis sp. Chlorella sp. e Isochrysis sp., para los cuales se ha reportado concentraciones de aceite hasta de $75 \%, 68 \%$, $56 \%$ y $33 \%$ de la biomasa seca, respectivamente [7], [11].

Durante el cultivo de las algas, el hierro se considera el micronutriente más importante, pues es vital para el funcionamiento del metabolismo, debido a su papel clave en el transporte de electrones, reducción de nitritos, nitratos y sulfatos, fijación del nitrógeno molecular y eliminación de especies reactivas como radicales libres y peróxidos [12].

Estudios previos [11], [12] demostraron el efecto de hierro en el crecimiento y en la acumulación de aceites en la microalga Chlorella vulgaris con agua de mar enriquecida. El mayor contenido de aceites obtenido fue $56,6 \%$ de la biomasa en base seca, a una concentración de $12 \mu \mathrm{M}$ de $\mathrm{Fe}^{3+}$ [11]. Cuando la concentración de $\mathrm{Fe}^{2+}$ es $17,92 \mu \mathrm{M}$, el contenido de aceites en base seca alcanzó un valor de 18,2\% [13]. Por otro lado, una concentración de 50,0 $\mu \mathrm{M} \mathrm{Fe}^{3+}$ no inhibe el crecimiento de la microalga Chlorella vulgaris [9]. Finalmente, Estévez et al. encontraron que con el empleo del medio BBM enriquecido con glucosa, la inhibición en la producción de biomasa no se alcanzó sino a concentraciones superiores a $200 \mu \mathrm{M}$ de Fe ${ }^{2+}$ [14].

Se ha estudiado el efecto del hierro en diferentes microalgas [15]-[19]. Yeesang y Cheirsilp determinaron el efecto del ión férrico presente en el medio heterotrófico Chu 13 en la producción de biomasa y acumulación de lípidos para cuatro cepas diferentes de Botryococcus braunii, aisladas de cuerpos de agua dulce del sur de Tailandia; las concentraciones óptimas para la producción de biomasa para las cepas PSU y SK fueron de 37 $\mu \mathrm{M}$ y $370 \mu \mathrm{M}$, respectivamente, mientras que el crecimiento de la cepa KB se inhibió a concentraciones superiores a $740 \mu \mathrm{M}$. Por su parte, la cepa TRG no experimentó cambios en la producción de biomasa con relación al hierro [15]. La gran disparidad de resultados demuestra la particularidad del efecto del hierro en cada cepa y refuerza la necesidad de evaluación experimental en cada caso.

El efecto del hierro también fue estudiado en las microalgas dinoflageladas Scrippsiella trochoidea y Alexandrium tamarense, conocidas por formar parte de las mareas rojas observadas en aguas marinas y responsables de daños ecológicos y económicos [16], [17]. Mientras que para la microalga Scrippsiella trochoidea se observó un efecto positivo en el crecimiento de la microalga para valores de $\mathrm{Fe}^{3+}$ entre 0,39 y $3,9 \mu \mathrm{M}$ y un efecto de saturación por dicho ión entre 3,9 y $39 \mu \mathrm{M}$ [16], para la microalga Alexandrium tamarense el efecto positivo del hierro fue evidenciado entre 1 $\mathrm{nM}$ y $1 \mu \mathrm{M}$ pero para concentraciones entre 1 y 10 uM la microalga experimentó inhibición [17].

Cai et al. evaluaron el efecto del empleo de los iones ferroso y férrico en la obtención de biomasa y astaxantina en la microalga Haematococcus pluvialis encontrando que el ión férrico favoreció el crecimiento de la microalga en mayor medida que el ión ferroso [18]. Por último, para la microalga Dunaliella tertiolecta se demostró que la eliminación del hierro en el medio de cultivo ocasiona un descenso en el crecimiento de la microalga mayor que el de cualquier otro nutriente [19].

El objetivo de este trabajo fue evaluar el efecto de la concentración del ión ferroso $\left(\mathrm{Fe}^{2+}\right)$ en la producción de biomasa y acumulación de lípidos de la cepa colombiana de microalga Chlorella vulgaris LAUN 0019, debido a su alta velocidad de crecimiento con respecto a otras cepas disponibles de microalgas nativas.

\section{MATERIALES Y MÉTODOS}

\section{A. Mantenimiento de la microalga}

Se utilizó la cepa de microalga nativa Chlorella vulgaris LAUN 0019, mantenida en el Laboratorio de Microalgas del Departamento de Biología de la Universidad Nacional de Colombia, sede Bogotá. 
La cepa fue incubada en cajas de Petri con medio Bristol estándar con la siguiente composición en mg.t-1: $\mathrm{NaNO}_{3} 250, \mathrm{KH}_{2} \mathrm{PO}_{4} 175, \mathrm{CaCl}_{2} \cdot \mathrm{H}_{2} \mathrm{O}$ $25, \mathrm{~K}_{2} \mathrm{HPO}_{4} 75, \mathrm{NaCl} 25, \mathrm{MgSO}_{4} \cdot 7 \mathrm{H}_{2} \mathrm{O} 75, \mathrm{H}_{3} \mathrm{BO}_{3}$ 11,42, $\mathrm{KOH} 31$, EDTA $50, \mathrm{FeSO}_{4} \cdot 7 \mathrm{H}_{2} \mathrm{O} 0,598$, $\mathrm{H}_{2} \mathrm{SO}_{4} 0,1, \mathrm{ZnSO}_{4} \cdot 7 \mathrm{H}_{2} \mathrm{O} 8,82, \mathrm{MnCl}_{2} \cdot 4 \mathrm{H}_{2} \mathrm{O} 1,44$, $\mathrm{MoO}_{3} \mathrm{O}, 71, \mathrm{CuSO}_{4} \cdot 5 \mathrm{H}_{2} \mathrm{O} 1,57$ y $\mathrm{Co}\left(\mathrm{NO}_{3}\right)_{2} \cdot 6 \mathrm{H}_{2} \mathrm{O}$ 0,49 . El medio de cultivo empleado en la caja de Petri se mantuvo sólido al agregar agar al $1 \%$. Las condiciones de cultivo incluyeron una temperatura de $23 \pm 1{ }^{\circ} \mathrm{C}$, iluminancia de $3550 \pm 20 \mathrm{~lx}$ y fotoperiodo de 16 horas de luz y 8 de oscuridad (16:8 L:0). Para la obtención del inóculo, la cepa fue transferida a un Erlenmeyer de $250 \mathrm{~mL}$ mediante lavado con medio Bristol líquido y cultivada durante 8 días en el mismo medio con un volumen de cultivo de $150 \mathrm{~mL}$ y burbujeo con aire atmosférico. El inóculo fue centrifugado en una centrífuga Hettich Zentrifugen tipo ROTOFIX 32 de $8 \mathrm{~cm}$ de radio y capacidad aproximada de 400 $\mathrm{mL}$, durante 25 minutos a $3500 \mathrm{rpm}(1097 \times \mathrm{g})$ y se descartó el sobrenadante. Las células fueron resuspendidas en un volumen pequeño de medio fresco para determinar la concentración celular y el volumen apropiado de medio fue transferido a cada recipiente para la evaluación experimental.

\section{B. Diseño experimental}

Para el estudio del efecto del hierro sobre el crecimiento de Chlorella vulgaris y su contenido de lípidos totales se analizaron cinco concentraciones diferentes de $\mathrm{FeSO}_{4} \cdot 7 \mathrm{H}_{2} \mathrm{O}$. La relación másica del $\mathrm{Fe}^{2+}$ y el EDTA en cada medio se mantuvo constante $\left(83,6 \mathrm{mg}\right.$ EDTA/mg FeSO $\left.\cdot 7 \mathrm{H}_{2} \mathrm{O}\right)$. Las concentraciones de $\mathrm{Fe}^{2+}$ fueron $2,16 \mu \mathrm{M}$, $5,00 \mu \mathrm{M}, 10,0 \mu \mathrm{M} 21,5 \mu \mathrm{M}$ y $50,0 \mu \mathrm{M}$, en donde 2,16 $\mu \mathrm{M}$ corresponde a la concentración estándar del medio Bristol; tales concentraciones siguieron un incremento logarítmico. Cada medio se evaluó por triplicado.

\section{Condiciones de cultivo}

La evaluación del efecto del hierro se realizó al cultivar la cepa en Erlenmeyers de $250 \mathrm{~mL}$ con medio Bristol enriquecido con $\mathrm{Fe}^{2+}$ como se describió en el diseño experimental. Las condiciones de crecimiento fueron: Volumen de medio de cultivo $150 \pm 2 \mathrm{~mL}$, densidad celular inicial: $~ 1 \times 10^{6}$ células $\cdot \mathrm{mL}^{-1}$; Temperatura $23 \pm 1$ ${ }^{\circ} \mathrm{C}$; 2 lámparas fluorescentes como fuente lumí- nica, iluminancia de $3770 \pm 20$ Ix y fotoperiodo de 16:8 L:0; burbujeo con aire atmosférico $\left(\mathrm{CO}_{2}\right.$ estimado en 350 ppm). El tiempo de cultivo fue 8 días.

\section{Medición de crecimiento}

El crecimiento en los diferentes medios de cultivo fue determinado mediante conteo celular diario con la cámara de Neubauer y microscopio LEICA DME.

\section{E. Rendimiento de peso seco}

Al finalizar el cultivo de las microalgas, las muestras se centrifugaron a $4000 \mathrm{rpm}$ durante 15 minutos $(1432 \times g)$ y se secaron en un horno de vacío (VWR Scientific), con un vacío de 20 in $\mathrm{Hg}$ a $60^{\circ} \mathrm{C}$ hasta peso constante (24 horas).

\section{F. Contenido de lípidos totales}

El contenido de lípidos totales se determinó por gravimetría mediante extracción por Soxhlet con Benzina de Petróleo como solvente, de acuerdo con el procedimiento estándar establecido por ASTM C 613C 613M-97 [20].

\section{G. Ajuste cinéticas de crecimiento}

A cada cultivo se ajustó una regresión a la fase exponencial de crecimiento según se muestra en (1).

$$
X=X_{0} e^{\mu t}
$$

Donde $X$ es la densidad celular células $\left.\mathrm{mL}^{-1}\right)$, $X_{0}$ es la densidad celular inicial (células $\cdot \mathrm{mL}^{-1}$ ), $\mu$ es la velocidad específica de crecimiento $\left(\mathrm{dia}^{-1}\right)$ y $t$ es el tiempo de cultivo (día). Para las regresiones se empleó Microsoft Excel ${ }^{\circledR}$.

Para la determinación del efecto del hierro en $\mu$ se ajustó el modelo de Monod con inhibición por sustrato, (2), en donde $\mathrm{S}$ es la concentración de hierro $(\mu \mathrm{M}), \mu_{\max }$ es la velocidad específica de crecimiento máxima sin inhibición

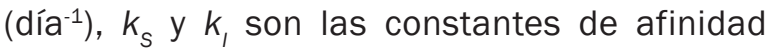
al sustrato $\left(\mathrm{Fe}^{2+}\right)$ e inhibición por sustrato respectivamente $(\mu \mathrm{M})$. Para el ajuste se empleó el software TableCurve 2D ${ }^{\circledR}$ (SYSTAT INC, San Jose, California, USA).

$$
\mu=\mu_{\max } \frac{S}{k_{s}+S+\frac{S^{2}}{k_{1}}}
$$




\section{H. Análisis de varianza}

Se realizó un análisis de varianza (ANAVA) a los datos obtenidos tanto de la productividad de biomasa como la de lípidos totales con la herramienta Análisis de datos de Microsoft Excel ${ }^{\circledR}$ con un nivel de significancia del $5 \%$.

\section{RESULTADOS Y DISCUSIÓN}

La Fig. 1 muestra el crecimiento de la microalga Chlorella vulgaris expresado como densidad celular en función del tiempo para las cinco concentraciones de hierro estudiadas. Cada punto corresponde al promedio de un triplicado. Para todas las concentraciones del ión ferroso estudiadas la fase de adaptación de la microalga no superó las 12 horas, mientras que la fase de crecimiento exponencial tomó alrededor de cuatro días del cultivo, por lo que la Ecuación (1) ajustó con coeficientes de determinación $\left(R^{2}\right)$ superiores a 0,94 . Estos tiempos son relativamente pequeños comparados con datos reportados para Chlorella vulgaris, lo cual probablemente se pueda asociar a las diferencias en las condiciones de crecimiento. Estévez et al. reportan tiempos de ocho días para la fase de adaptación y siete días para la fase exponencial [14]; la extensión en la fase de adaptación posiblemente esté asociada a que el precultivo de la microalga fue llevado a cabo en medio sólido, mientras que en nuestro caso el inóculo provino a un medio muy similar al medio de prueba. La mayor duración de la fase exponencial pudo deberse al empleo de la glucosa en lugar de $\mathrm{CO}_{2}$ como fuente de carbono, convirtiendo de esta manera el medio de cultivo BBM, muy similar al medio Bristol, de autotrófico a heterotrófico [14]. Por su parte, Liu et al. reportaron un crecimiento final similar al obtenido, aunque con una duración de la fase exponencial mucho más prolongada (21 días) [11], lo que implica que la velocidad de crecimiento en este artículo fue mucho menor, la probable causa del lento crecimiento reportado por Liu et al. pudo ser el empleo de $f / 2$ como medio de cultivo, dado que éste es un medio marino de elevada salinidad, la cual habría reducido la velocidad de crecimiento del alga que se empleó en dicha investigación. Un fenómeno similar fue reportado por Cho et al., quienes demostraron que la mayor velocidad de crecimiento y biomasa final de Chlorella ellipsoidea se obtenía a la menor concentración salina probada (10 g.L $\left.\mathrm{L}^{-1}\right)$ [21].

FIG. 1. EFECTO DEL IÓN FE ${ }^{2+}$ EN EL CRECIMIENTO DE CHLORELLA VULGARIS

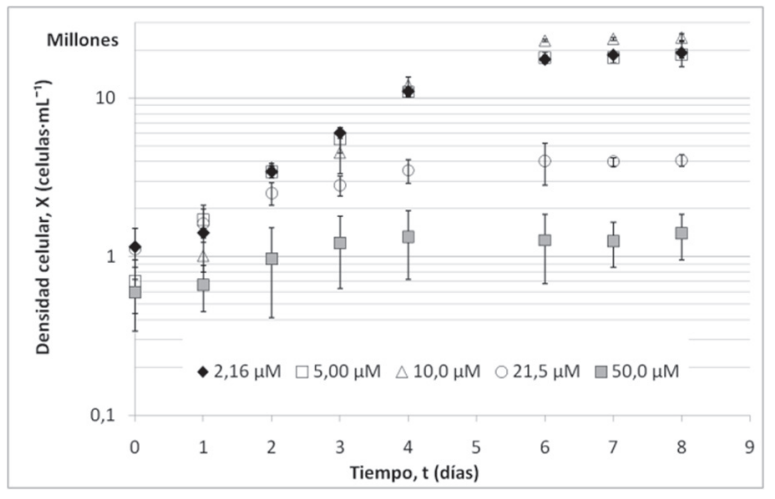

La máxima densidad celular encontrada al finalizar el cultivo, $2,4 \times 10^{7}$ células-mL $\mathrm{L}^{-1}$, se observó a una concentración experimental de hierro de $10,0 \mu \mathrm{M}$, en promedio fue un $25,8 \%$ mayor que las densidades de las concentraciones de hierro inferiores probadas, las cuales son muy similares entre sí con una dispersión de apenas el 2,4\%; por otro lado se aprecia una reducción en las densidad celular final para las concentraciones 21,5 y $50,0 \mu \mathrm{M}$.

La Fig. 2 muestra el valor de $\mu$ ajustado durante la fase exponencial en función de la concentración de hierro; se evidencia un máximo para una concentración de 10,0 $\mu \mathrm{M}$ y posteriormente un descenso para las concentraciones de 21,5 y $50,0 \mu \mathrm{M}$. Debido a que para la máxima concentración de hierro, éste representó sólo el $2 \%$ de la salinidad total del cultivo se concluye que el decaimiento de $\mu$ fue causado por una inhibición por exceso de sustrato, y no por el posible estrés que pudo experimentar la microalga al ser sometida a un aumento de la fuerza iónica al incrementar la concentración de hierro.

Se ajustó una cinética de Monod con inhibición por sustrato a los datos experimentales, obteniéndose un coeficiente de determinación de 0,92 . El modelo predice un $\mu$ óptimo para una concentración de hierro de 5,24 $\mu \mathrm{M}$; a pesar del buen ajuste del modelo a los datos, es necesario señalar que la aparente reducción en la velocidad de crecimiento a mayores concentraciones de hierro pudo ser resultado indirecto 
de una mayor concentración de EDTA, el cual posiblemente queló algún otro ión necesario para el crecimiento celular, sin embargo, en la experimentación seguida por Estévez et. al., la relación EDTA - hierro se mantuvo constante en 2:1, al emplear como base el contenido de EDTA en el medio BBM [14], el cual es el mismo que en el medio Bristol, y como se mencionó previamente la inhibición por sustrato no se observó hasta después de una concentración de 200 $\mu \mathrm{M}$, por lo cual es posible concluir que la inhibición no fue causada por el EDTA.

FIG. 2. EFECTO DEL IÓN FE ${ }^{2+}$ EN LA VELOCIDAD ESPECÍFICA DE CRECIMIENTO DE CHLORELLA VULGARIS DURANTE FASE DE CRECIMIENTO EXPONENCIAL

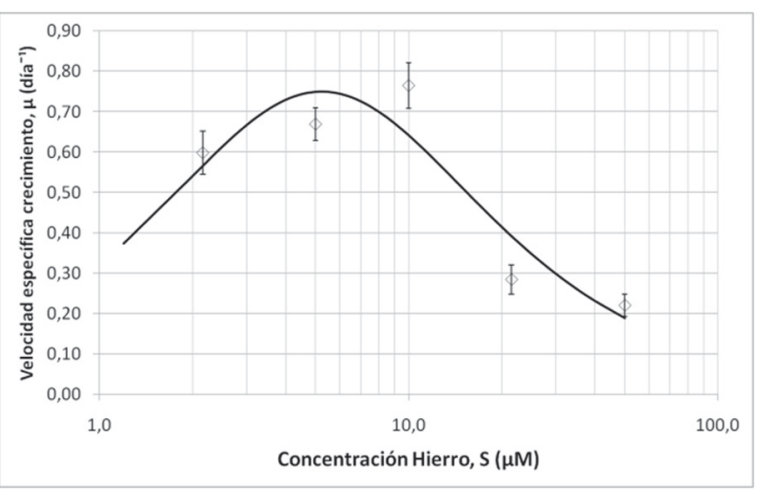

El efecto del hierro sobre la productividad de biomasa de Chlorella vulgaris se presenta en la Fig. 3. La productividad fue calculada para el último día de cultivo al dividir el peso seco de la biomasa final entre el tiempo de cultivo ( 8 días) y el volumen de cultivo (150mL). Para los estudios de productividad se descartó el ensayo con concentración de hierro 50,0 $\mu \mathrm{M}$ debido a que su densidad celular fue extremadamente baja, como se aprecia en la Fig. 1. En la Fig. 3 se observa un máximo entre $5,00 \mu \mathrm{M}$ y 10,00 $\mu \mathrm{M}$, lo cual coincide con la Fig. 2. Según el ANAVA mostrado en la Tabla I, la concentración de hierro sí incidió en la productividad de biomasa con una confiabilidad del 95\%; sin embargo, al realizar un nuevo ANAVA, Tabla II, pero esta vez sólo para los ensayos previos al óptimo experimental $(2,15,5,00$ y $\quad 10,0 \mu \mathrm{M})$ se concluyó que el hierro no afectó de manera significativa la productividad de biomasa a dichas concentraciones de hierro con una confiabilidad del 95\%, aunque al reducir la confiabilidad al 94\% la conclusión es opuesta.

FIG. 3. EFECTO DEL IÓN FE ${ }^{2+}$ SOBRE LA PRODUCTIVIDAD DE BIOMASA DE CHLORELLA VULGARIS DESPUÉS DE 8 DÍAS DE CULTIVO

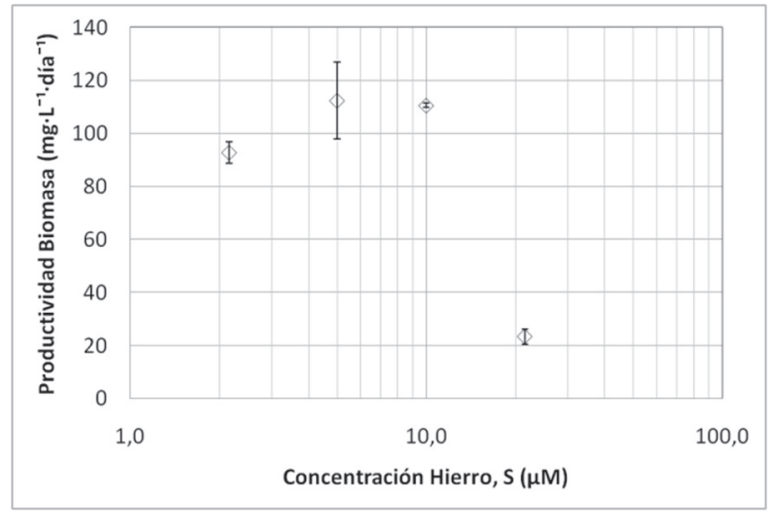

Tabla I.

ANAVA efecto hierro en productividad de biomasa (S: 2,15; 5,00; 10,0; $21,5 \mu \mathrm{M}$ )

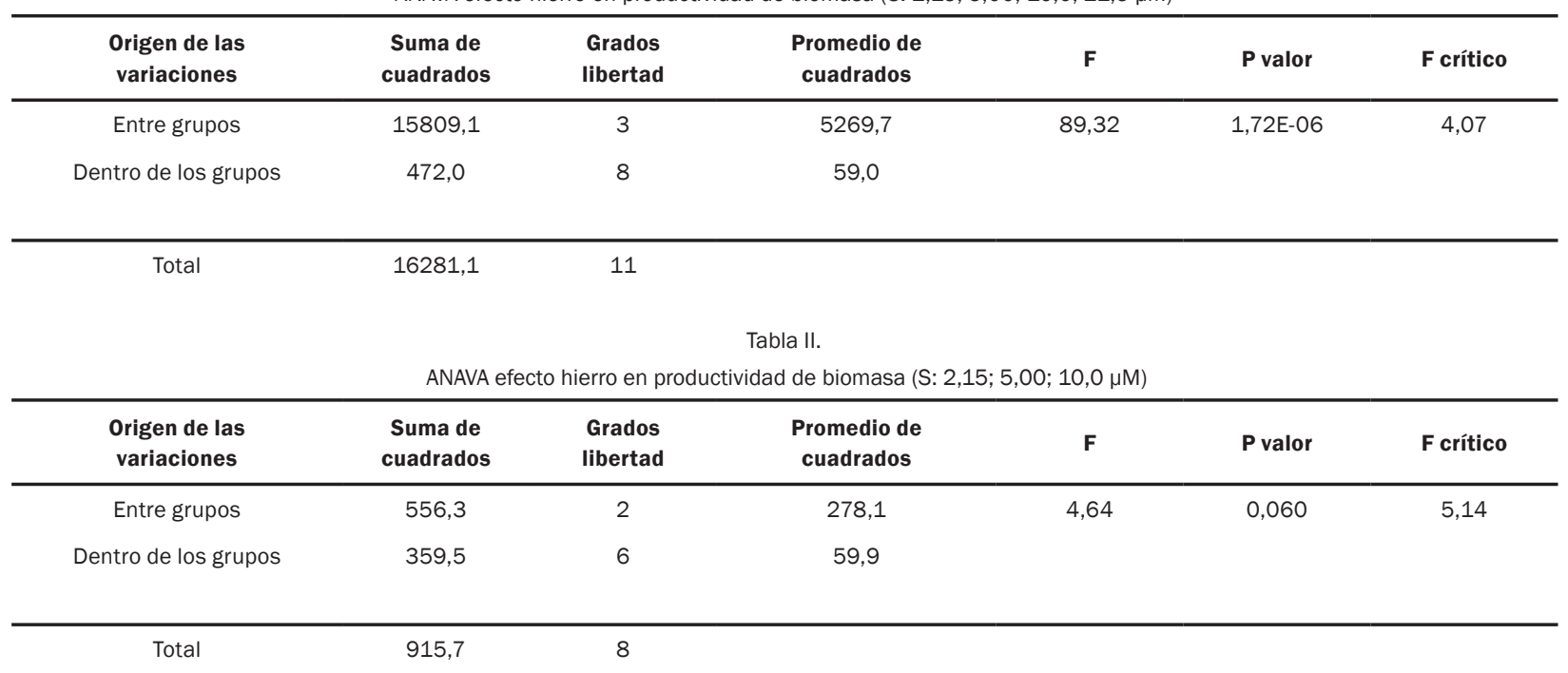


De acuerdo con la Fig. 4, la máxima acumulación de lípidos en la microalga seca ocurrió cuando se empleó el medio estándar $(2,16 \mu \mathrm{M}$ de $\mathrm{Fe}^{2+}$ ), con un valor de $6,5 \%$. Sin embargo, si se considera la productividad de lípidos después de los ocho días de cultivo, para una concentración de hierro de 5,00 $\mu \mathrm{M}$ se presenta un máximo de 6,52 $\mathrm{mg} \cdot \mathrm{L}^{-1} \cdot \mathrm{di}^{-1}{ }^{-1}$ como lo demuestra la Fig. 5. Por otro lado, según los resultados estadísticos, Tabla III, la concentración del hierro no influencia significativamente la productividad de lípidos totales, por lo cual, para una eventual producción a gran escala lo más conveniente es escoger bajas concentraciones de hierro para una reducción de los costos en las materias primas.

FIG. 4. EFECTO DEL IÓN FE ${ }^{2+}$ SOBRE EL CONTENIDO DE LÍPIDOS EN CHLORELLA VULGARIS DESPUÉS DE 8 DÍAS DE CULTIVO

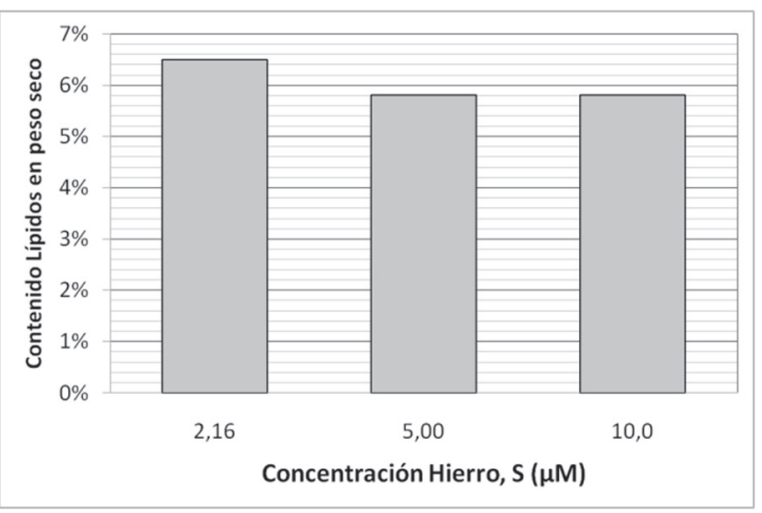

La acumulación de lípidos encontrada es baja independiente de la concentración de hierro utilizada, comparada con la reportada en otras investigaciones en la literatura, pues como se mencionó previamente Liu et al. reportan porcentajes de aceites superiores al 56\% [11], esto posiblemente ocasionado por la salinidad, ya que el estrés causado por ésta pudo inducir a la microalga a acumular lípidos en gran cantidad, pues en investigaciones previas se demostró el efecto positivo de la salinidad en la acumulación de lípidos en la microalga Botryococcus braunii [8], [22]; otra causa podría ser la valencia del hierro empleado, ya que Liu et al. emplearon el ión férrico mientras que el presente estudio utilizó el ión ferroso como fuente de hierro, ya que para la microalga Haematococcus pluvialis, como se refirió previamente, el ión férrico favoreció la producción de lípidos en mayor proporción que el ión ferroso [18], dichos efectos de la salinidad y la valencia podrían ser equivalentes para la microalga Chlorella vulgaris.

FIG. 5. EFECTO DEL IÓN FE ${ }^{2+}$ SOBRE LA PRODUCTIVIDAD DE

LIIPIDOS EN CHLORELLA VULGARIS DESPUÉS DE 8 DÍAS DE CULTIVO

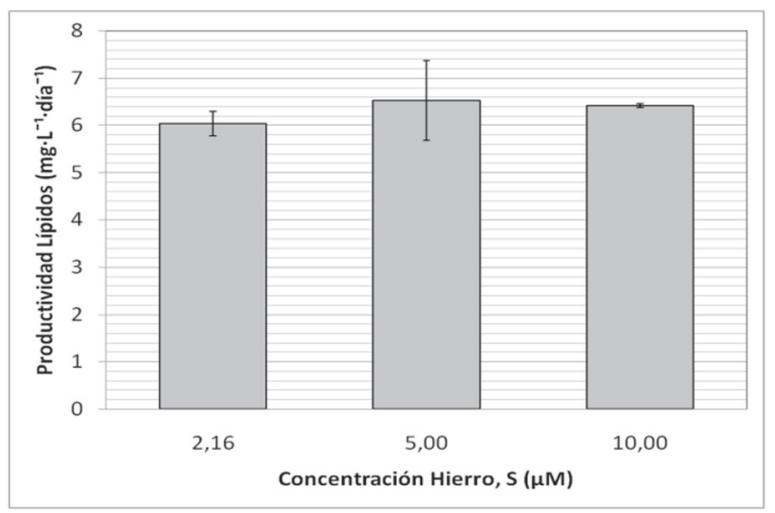

Es muy probable que la acumulación de lípidos tenga una mayor dependencia de la concentración de $\mathrm{CO}_{2}$ en el aire que de la concentración de hierro en el medio, por lo cual es necesario desarrollar trabajos para comprobar tal hipótesis.

Finalmente, el porcentaje de lípidos encontrado no tiene los niveles necesarios para un escalamiento a nivel industrial y pueda competir con el aceite de palma africana, pues para la suposición de un cultivo por lotes de la microalga durante 5 días con tiempo muerto de otros 2 días en estanques abiertos de $30 \mathrm{~cm}$ de profundidad, la productividad de lípidos equivaldría máximo a 5,1 ton'ha ${ }^{-1} \cdot a^{n} 0^{-1}$, valor muy similar al reportado para la palma africana [7], sin embargo, no tiene en cuenta la posible reducción en la productividad de biomasa ocasionada por el escalamiento del cultivo ni la eficiencia en el empleo de métodos industriales para la extracción de lípidos, por lo que es necesario continuar con las investigaciones para buscar aumentos en la composición de lípidos en la microalga.

Tabla III.

ANAVA efecto hierro en productividad de lípidos (S: 2,15; 5,00; 10,0 $4 \mathrm{M}$ )

\begin{tabular}{cccccc}
\hline $\begin{array}{c}\text { Origen de las } \\
\text { variaciones }\end{array}$ & $\begin{array}{c}\text { Suma de } \\
\text { cuadrados }\end{array}$ & $\begin{array}{c}\text { Grados } \\
\text { libertad }\end{array}$ & $\begin{array}{c}\text { Promedio de } \\
\text { cuadrados }\end{array}$ & F crítico & P valor \\
\hline Entre grupos & 0,39 & 2 & 0,197 & 0,759 & 0,508 \\
Dentro de los grupos & 1,56 & 6 & 0,260 & \\
\hline Total & 1,95 & 8 &
\end{tabular}




\section{CONCLUSIÓN}

Se utilizó un modelo cinético de crecimiento de Monod con inhibición para explicar el efecto del hierro en el crecimiento y la acumulación de lípidos en la microalga Chlorella vulgaris. El modelo ajustó los datos experimentales con un coeficiente de determinación de 0,92. La máxima velocidad específica de crecimiento fue de 0,76 dia $^{-1}$ para una concentración de 10,0 $\mu \mathrm{M}$, lo que en otras palabras significa un tiempo de duplicación de la microalga mínimo de 0,91 días. Aunque en este trabajo se atribuye el efecto de inhibición observado a la alta concentración de hierro, tal efecto bien podría deberse al incremento correspondiente en la concentración de EDTA (acomplejando algún otro ión, reduciendo su disponibilidad) o al efecto combinado $\mathrm{Fe}^{2+}$ - EDTA. Liu et al. [11] mantuvieron constante la relación $\mathrm{Fe}^{3+}$ - EDTA durante su diseño experimental sin encontrar inhibición alguna, mientras que Estévez et al. [14] también mantuvieron contante esta relación y la inhibición se observó después de $200 \mu \mathrm{M}$. Para resolver este interrogante, se sugiere que en futuros trabajos se realice un nuevo diseño experimental donde se pueda determinar dicho efecto.

El rendimiento de alga en peso seco fue 0,88 g. $L^{-1}$ para una concentración de hierro de $5,00 \mu \mathrm{M}$ después de 8 días de cultivo y la concentración estimada de hierro más favorable para la acumulación de lípidos durante el crecimiento de la microalga fue de $2,16 \mu \mathrm{M}$, se obtuvo un contenido de lípidos de $6,5 \%$ del peso seco a escala laboratorio.

El análisis estadístico mostró que a concentraciones bajas de hierro, éste no tuvo un efecto significativo sobre la productividad de lípidos en las condiciones estudiadas para la cepa colombiana, pero no puede generalizarse este resultado ya que como se puede concluir de la investigación de Yeesang y Cheirsilp, el efecto del hierro en el crecimiento y acumulación de lípidos es característico en cada cepa [15].

\section{AGRADECIMIENTOS}

Los autores agradecen al Laboratorio de Microalgas del Departamento de Biología de la Universidad Nacional de Colombia sede Bogotá por el suministro de la cepa LAUN 0019 y a su director, el biólogo Luis Carlos Montenegro.

\section{REFERENCIAS}

[1] COMPES 3510 "Lineamientos de política para promover la producción sostenible de biocombustibles en Colombia". Bogotá: Departamento Nacional de Planeación, 2008.

[2] C. Razo, S. Astete Miller, A. Saucedo, and C. Ludeña, "Biocombustibles y su impacto potencial en la estructura agraria, precios y empleo en América Latina", División de Desarrollo Productivo y Empresarial CEPAL. Santiago de Chile. Serie Desarrollo Productivo No 178, 2007

[3] C. M. Drapcho, N. P. Nhuan, and T. H. Walker, Biofuels Engineering Process Technology, vol. I., New York: McGraw Hill, 2008

[4] Fedepalma, "Oferta y consumo aparente mundial de los 17 principales aceites y grasas". http://www.fedepalma.org/documen/2008/ consumo_mundial.pdf consultado 3 de Octubre de 2009

[5] FAO. "Food and Agricultural commodities production", http:// faostat.fao.org/site/339/default. aspx consultado el Noviembre 06, 2010

[6] E. P. Feofilova, Y. E. Sergeeva, and A.A. Ivashechkin, "Biodiesel-fuel: Content, production, producers, contemporary biotechnology (Review)", Applied Biochemistry and Microbiology, vol. 46, no. 4, pp. 369-378, 2010

[7] Y. Chisti, "Biodiesel from microalgae", Biotechnology Advances, vol. 25, no. 3, pp. 294-306, 2007

[8] A. Banerjee, R. Sharma and Y. Chisti, "Botryococcus braunii: A Renewable Source of Hydrocarbons and Other Chemicals", Critical Reviews in Biotechnology, vol. 22, no. 3, pp. 245-279, 2002

[9] G.Petkov and G.Garcia, "Which are fatty acids of the green alga Chlorella?", Biochemical Systematics and Ecology, vol. 35, no. 5, pp. 281-285, 2007

[10] Acosta,. "Biocombustibles: Oportunidad o Amenaza". Presented at the Seminario Internacional De Biocombustibles 2009 CORPODIB. Bogotá, Colombia, Febrero 12, 2009

[11] Z. Y. Liu, G. C. Wang and B. C. Zhou, "Effect of iron on growth and lipid accumulation in ChloreIla vulgaris", Bioresource Technology, vol. 99, no. 11, pp. 4717-4722, 2008 
[12] R. Andersen, Algal culturing techniques, vol. I. China: Elsevier Inc., 2005

[13] F. Cleber Bertoldi, E. Sant'Anna, M. V. da Costa Braga and J. L. Barcelos Oliveira, "Lipids, fatty acids composition and carotenoids of Chlorella vulgaris cultivated", Grasas y Aceites, vol. 57, no.3, pp. 270-274, 2006

[14] M. S. Estevez, G. Malanga and S. Puntarulo. "Iron-dependent oxidative stress in Chlorella vulgaris", Plant Science, vol. 161, no.1, pp. 9-17, 2001.

[15] C. Yeesang and B. Cheirsilp, "Effect of nitrogen, salt, and iron content in the growth medium and light intensity on lipid production by microalgae isolated from freshwater sources in Thailand", Bioresource Technology, vol. 102, no.3, pp. 3034-3040, 2011

[16] C. Zhuo-Ping, H. Wei-Wei, A. Min and D. ShunShan, "Coupled effects of irradiance and iron on the growth of a harmful algal bloom-causing microalga Scrippsiella trochoidea", Acta Ecologica Sinica, vol. 29, no.5, pp. 297-301, 2009

[17] H. He, F. Chen, H. Li, W. Xiang, Y. Li and Y. Jiang, "Effect of iron on growth, biochemical composition and paralytic shellfish poisoning toxins production of Alexandrium tamarense", Harmful Algae, vol. 9, no.1, pp. 98-104, 2010

[18] M. Cai, A. Qi, Y. Zhang and Z. Li, “Effects of iron electrovalence and species on growth of Haematococcus pluvialis and astaxanthin production", Journal of Biotechnology, vol. 136, no.1, pp. S574, 2008

[19] M. Chen, H. Tang, H. Ma, T. C. Holland, K.Y. Simon $\mathrm{Ng}$ and S. O. Salley, "Effect of nutrients on growth and lipid accumulation in the green algae Dunaliella tertiolecta", Bioresource Technology, vol. 102, no.2, pp. 1649-1655, 2011

[20] ASTM International. ASTM C 613/C 613M - 97. Standard Test Method for Constituent Content of Composite Prepreg by Soxhlet Extraction, 2008

[21] S. H. Cho, S.C. Ji, S. B. Hur, J. Bae, I.S. Park, Y. C. Song, "Optimum temperature and salinity conditions for growth of green algae Chlorella ellipsoidea and Nannochloris oculata", Fisheries Science, vol. 73, no.5, pp. 1050-1056, 2007

[22] A. Ranga Rao, C. Dayananda, R. Sarada, T.R. Shamala and G.A. Ravishankar, "Effect of salini- ty on growth of green alga Botryococcus braunii and its constituents", Bioresource Technology, vol. 98, no.3, pp. 560-564, 2007 